\title{
A Study of Ki 67 Immunostaining in Prostate Carcinomas: Correlation with Gleason's Score
}

\author{
Roopa Urs A.N ${ }^{1 *}$, Suchitha $\mathrm{S}^{2}$, Manjunath $\mathrm{GV}^{2}$ and Hugara Siddalingappa ${ }^{3}$ \\ ${ }^{1}$ Department of Pathology, Mahatma Gandhi Medical College and Research Institute, Puducherry, India \\ ${ }^{2}$ Department of Pathology, JSS Medical College, Mysore, India \\ ${ }^{3}$ Department of Community Medicine, Mandya Institute of Medical Sciences, Mandya, India
}

\begin{abstract}
Background: One of the most common causes of carcinoma deaths among men is prostate cancer. In view of the above, early diagnosis and effective treatment of the disease are immensely important. The increasing number of options for the treatment of prostate cancer has made the prognostic evaluation of the disease even more important. Proliferation plays an important role in the clinical behaviour of prostate cancer. $\mathrm{Ki}-67$ binding, is an objective measurement of cell proliferation which significantly aids in the management of the prostate patients. Gleason grading describes the aggressiveness of prostate cancer. The Objectives was to study the immunostaining patterns of Ki-67 in prostate cancers and to compare the results with Gleason's score.
\end{abstract}

Methods: Fifty cases of histopathologically proven prostate carcinomas diagnosed on needle biopsies and transurethral resection specimens was studied in JSS medical college and hospital, mysore for a period of 3years and histopathological grade was assessed using Gleason grading system. Immunohistochemistry (IHC) for Ki-67 was done on paraffin embedded wax sections.

Result: Ki-67 was positive in $49 / 50$ cases (98\%). The range of Ki-67 score was 0 to $94 \%$. No statistically significant association was seen with Ki-67 and gleasons scores $(\mathrm{P}=0.277)$.

Conclusion: Proliferation has been recognized as a distinct hallmark of cancer and acts as an important determinant of cancer outcome. As $\mathrm{Ki}-67$ can be used to objectively measure this, it can be included in the pool of prognostic markers like tumor volume, histopathological grade and surgical margins.

Keywords: Prostate cancer, Ki -67, Gleason's score

\section{Introduction}

Prostate cancer is the second most common cause of cancer and the sixth leading cause of cancer death among men worldwide. Prostate cancer mortality is strongly related to age, with the highest mortality rates being in older males. Prostate cancer mortality rates are highest in the Caribbean and lowest in South Central Asia, but this partly reflects varying data quality worldwide. ${ }^{[1]}$

Patients generally do not experience symptoms during early stage and are unlikely to seek medical help until the disease has progressed. Thus, prostate cancer is acknowledged as a major health problem and with the advent of screening tests like digital rectal examination and prostate specific antigen (PSA) test, more patients are being diagnosed in earlier stages. With delay in early diagnosis of the lowgrade tumor, the quality or length of patient's life is not significantly changed, but a high-grade tumor in a young person might spread quickly and lead to the patient's death within two years. ${ }^{[2]}$

Prognostic factors in prostatic cancer are divided into clinical and biological groups. Laboratory investigations like blood tests, radiological and microscopic evaluation of biopsies are done to obtain clinical prognostic factors. Tumor grading is one of the important factors among them. Several grading systems have been introduced for prostate cancer. One of the method, which is very acceptable, is Gleason's grading. This method of is based on the patterns of tumor cell differentiation. The other category of prognostic importance are the biological prognostic factors in which p53, p27, and Ki-67 are important. ${ }^{[2]}$

Gleason system is the one recommended by World health organization and accepted by majority of urologists and radiotherapists. The Gleason grading system, named after Donald F. Gleason, is a unique histopathological method for grading prostate cancer based solely on the tumor architecture. ${ }^{[3,4]}$ It is important preoperative predictor of the behavior of prostate cancer and is used to help in making decisions about treatment for localized prostate cancer. ${ }^{[5,6]}$ It is also used to predict relapse in patients receiving hormone therapy for bone metastasis of prostate cancer. ${ }^{7}$

Proliferation plays an important role in the clinical behavior of prostate cancer. Increased proliferation correlates 
strongly with tumor aggressiveness and poor prognosis. ${ }^{[4]}$ However, of the different methods to assess proliferation, mitosis counting has been shown most convincingly to provide reproducible and independent prognostic value in prostate adenocarcinoma. Ki-67 binding, as an objective measurement of cell proliferation aids significantly in the management of the prostate cancer. Ki-67 index is higher in carcinoma than hyperplasia and still higher in metastatic than nonmetastatic cases, thus an increased Ki-67 index may indicate a poor prognosis of disease. ${ }^{[2]}$ However, its role as an independent prognostic marker among patients with prostate carcinoma is still controversial.

Despite improvements in early detection of prostate cancer as a result of PSA screening, we still lack molecular markers to effectively distinguish patients with high risk of disease progression from the indolent majority. Considering the proven correlation between Gleason's grading and prognosis of prostate cancer, $\mathrm{Ki}-67$ study is undertaken to investigate the frequency of expression of these markers in prostate cancer and their probable relation with Gleason's score.

\section{Materials and Methods}

In the present study, fifty cases of Prostate carcinomas diagnosed on needle biopsies and transurethral resection specimens were studied in JSS medical college and hospital, Mysore for a period of 3years. Ethical committee clearance was obtained. All surgically removed prostate carcinoma tissues was fixed in formalin followed by paraffin embedding and staining with haematoxylin and eosin. Sections were studied to evaluate histologic type and histologic grade. Histopathological grade was assessed using Gleason's grading system.

IHC for Ki-67 was done on $4 \mu \mathrm{m}$ thick paraffin embedded wax sections on poly-1 lysine coated slides. Antigen retrieval was done in tri sodium citrate buffer at $\mathrm{pH}$ 6. Monoclonal antibody Ki-67 (clone MM1) Novocastra code no Ki-67MM1-R7-C was used for Ki-67 antigen detection by one step horseradish peroxidase (HRP) polymer method. A section from a reactive lymph node was taken as positive control whereas sections treated with tris-buffer solution instead of primary antibody was used as negative control.

Brown granular nuclear reactivity was taken as positive. Ki-67 Labeling index (LI) was expressed as percentage of positively stained cells per 100 epithelial cells after counting at least 1000 cells using high power (40x10). An area with the maximum proliferation was chosen to evaluate the labeling index (LI).. Labeling Index for Ki67 was categorized as $\leq 2 \%$ - Negative, $<25 \%-1+, 26-50 \%$ $2+, 51-75 \%-3+, 76-100 \%-4+$. LI was correlated with histopathological grade.
Data was analysed using Epi-info statistical software. Univariate analysis was done and expressed in mean and percentages. Bivariate analysis was done using Spearman's correlation coefficient, to find out the strength of association between histopathological report results and Gleason's score. The association of $\mathrm{Ki}-67$ as a categorical variable was determined by chi square test. Results were interpreted at alpha error level of $5 \%$. p value of $<0.05$ was considered as statistically significant.

\section{Result}

In the present study, the age group of patients ranged from 44 to 86 years, with a mean age of 69.9 years. Patients predominantly presented with acute urinary retention (AUR) \& lower urinary tract symptoms (LUTS). All cases displayed features of Acinar Adenocarcinoma (Ordinary type) of which glandular pattern (84\%), followed by cribriform (56\%) were common patterns. In the present study, majority $(70 \%)$ had poorly differentiated adenocarcinoma followed by moderatelypoorly differentiated (24\%)[Table-1] No cases of well differentiated adenocarcinoma was encountered during the study period. Perineural invasion (PNI) was seen in $48 \%$ of the cases. One case (2\%) showed metastasis to bone which was picked up by Positron Emission Tomography (PET).

In Ki-67 stained samples, thirty four cases (68\%) were positive of which twenty six were poorly differentiated and eight cases were moderately differentiated and sixteen $(32 \%)$ cases stained negative. Twenty five $(50 \%)$ cases were graded as $1+(<25 \%)$ of which seventeen were poorly differentiated and eight were moderately differentiated. Six $(12 \%)$ cases were $2+(26-50 \%)$ and all were poorly differntiated, Two (4\%) cases were $3+(51-75 \%)$ and both were poorly differentiated [Figure-1,2] and one (2\%) poorly differentiated tumor had $4+(76-100 \%)$. Of eight cases of moderately differentiated tumors all were indexed as $1+$. No well differentiated cases were seen in this study. (Table-1, Figure-3)

\section{Discussion}

Majority $(70 \%)$ of tumors were poorly differentiated adenocarcinoma followed by moderately differentiated adenocarcinomas $(30 \%)$ in our study. Whereas, similar studies done by Madani SH et $\mathrm{al}^{[2]}(51 \%)$, Shirley SE et $\mathrm{al}^{[8]}(60 \%)$, Catalona WJ et $\mathrm{a}^{[10]}(60 \%)$ have also reported majority of adenocarcinoma with poorly differentiation having Gleason score between 8-10.

Ki-67 immunohistochemical marker was positive in $34(68 \%)$ cases out of 50 cases of prostate cancers. Majority (54\%) of positive stained Ki-67 cases were poorly differentiated tumors, few (14\%) were moderately differentiated tumors. No well differentiated tumor was 
Table 1: Frequency of Ki-67 labelling index in relation to differentiation and Gleason grade.

\begin{tabular}{|c|c|c|c|c|}
\hline \multirow{2}{*}{ Ki67 } & \multicolumn{2}{|c|}{ Gleason Grade } & Frequency & Percentage (\%) \\
\cline { 2 - 5 } & Moderately differentiated & Poorly differentiated & 16 & 32.0 \\
\hline$\leq 2 \%$ & 7 & 9 & 25 & 50.0 \\
\hline $26-50 \%$ & 8 & 17 & 6 & 12.0 \\
\hline $51-75 \%$ & 0 & 6 & 2 & 4.0 \\
\hline $76-100 \%$ & 0 & 2 & 1 & 2.0 \\
\hline Total & 0 & 1 & $\mathbf{5 0}$ & $\mathbf{1 0 0 . 0}$ \\
\hline
\end{tabular}

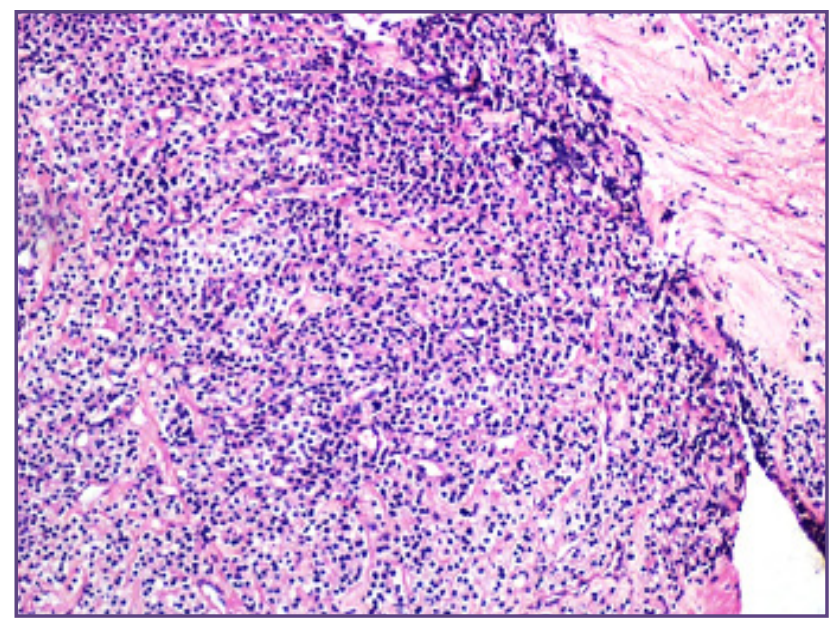

Fig. 1: Photomicrograph showing poorly differentiated adenocarcinoma with tumor cells arranged in sheets. Gleason's score 5+4=9 (H \& E x100).

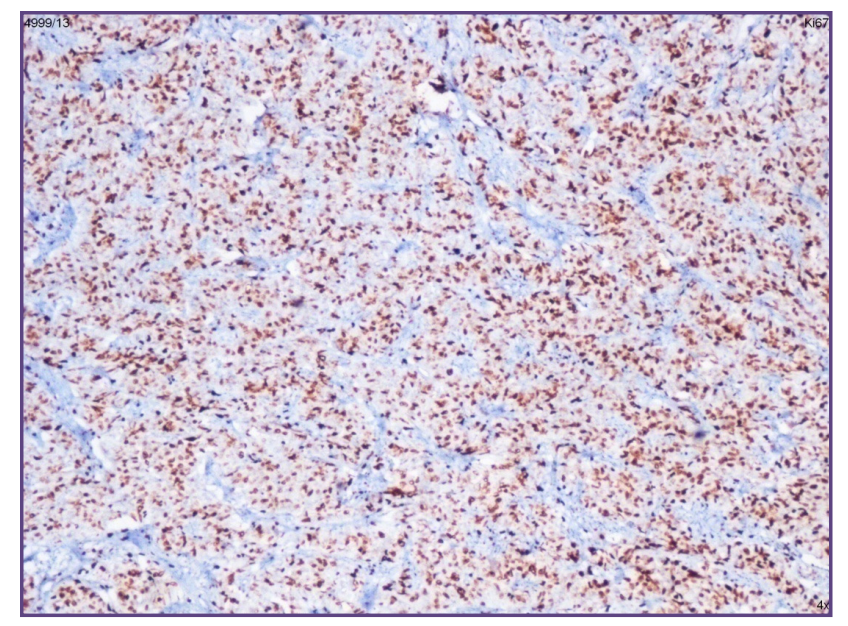

Fig. 2: Ki-67 staining in poorly differentiated prostatic adenocarcinoma with $3+$ positivity. Gleason's score 5+4=9 (Ki67, x40).

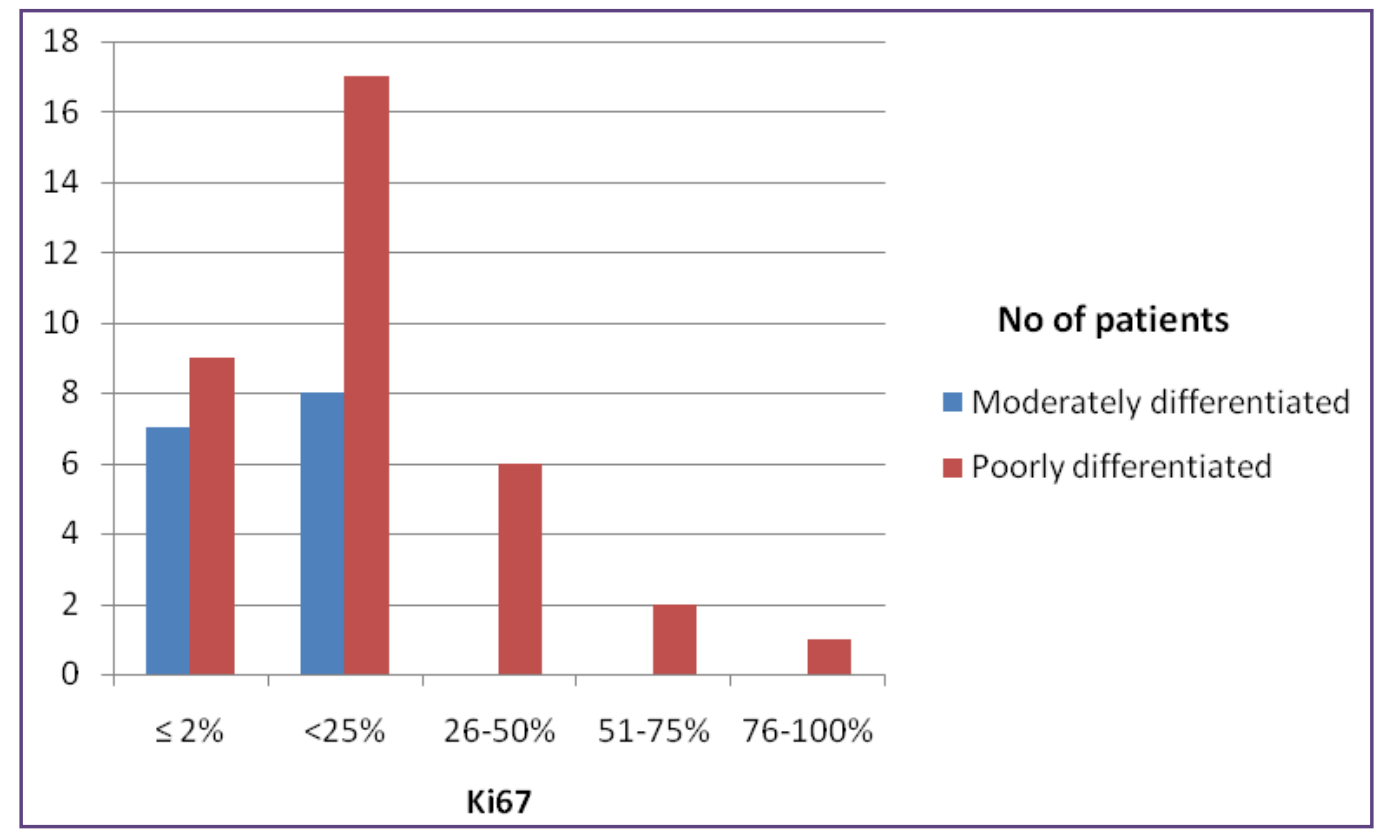

Fig. 3: Comparision of results of Ki67 and Gleason's score among study samples. 
seen. Studies done by Madani SH et $\mathrm{al}^{2}$, E.Munoz et $\mathrm{al}^{10}$, Rashed HE et $\mathrm{al}^{11}$, have shown increased Ki-67 positive cases with poorly differentiated tumors.

Although there was a comparative positive Ki-67 staining with increased Gleason's score, our study did not show statistical significance ('p' value $=0.277$ ). However, studies done by Theodoropoulos et $\mathrm{al}^{12}$, Nilson et $\mathrm{al}^{13}$, Madani SH et $\mathrm{al}^{2}$, Rashed HE et $\mathrm{al}^{11}$, Murti $\mathrm{K}{ }^{14}$, have shown a statistically significant relationship between Ki-67 index and Gleason's score of tumors with low- to high-grade differentiation and also important relationship with the prognosis of prostate cancer. In contrast, studies done by $\mathrm{E}$ Munoz et $\mathrm{al}^{10}$ and Thompson et al ${ }^{15}$ have not shown statistical correlation between Ki-67 and Gleason's score. But these studies have concluded that Ki-67 staining may allow identification of tumors with a high rate of cell growth and one of the important prognosticator which was consistent with our present study.

Multiple other studies have demonstrated that mean proliferation indices as measured by $\mathrm{Ki}-67$ staining are associated with disease progression, stage, Gleason's score, mean pretreatment prostate-specific antigen level and increase from benign to malignant tissue. These investigations support findings with proliferation index and may serve as a biomarker in future studies that compare biopsy specimens over time (i.e., neoadjuvant studies) or multiple biopsy specimens in the setting of chemoprevention.

\section{Conclusion}

To conclude, Ki-67 staining is one of the convenient and rapid method for assessing the proliferation and also more reproducible than other proliferative markers. Proliferative activity determined by Ki-67 may reflect the aggressive behavior of prostate cancer and predict the time of recurrence and the appropriate therapy. Ki67 can be used as prognostic indicator and further studies with more number of cases are required to determine their biologic role and progression of prostate cancer.

\section{Reference}

1. Ferlay J, Foucher ES, Tieulent LJ, Rosso S. Cancer incidence and mortality patterns in Europe: Estimates for 40 countries in 2012. European Journal of Cancer 2013;49:1374- 1403

2. Madani SH, Ameli S, Khazaei S, Kanani M, Izadi B. Frequency of Ki-67 (MIB-1) and P53 expressions among patients with prostate cancer. Indian J Pathol Microbiol 2011;54:688-91

3. Gleason DF, Mellinger GT. Prediction of prognosis for prostatic adenocarcinoma by combined histological grading and clinical staging. J Urol 1974; 111: 58-64.

4. Gleason DF. Classification of prostatic carcinomas. Cancer Chemother Rep. Part 1. 1966; 50(3): 125-8.

5. Mellinger GT, Gleason D, Bailar J. The histology and prognosis of prostatic cancer. J Urol 1967; 97(2):331-7.

6. Poulos CK, Daggy JK, Cheng L. Preoperative prediction of Gleason grade in radical prostatectomy specimens: the influence of different Gleason grades from multiple positive biopsy sites. Mod Pathol 2005; 18: 228-34.

7. Tsukamoto T, Kumamoto Y, Umehara T, Takahashi A, Shimazaki J, Ohshima $\mathrm{H}$ et al. Clinical study of bone-related relapse in prostate carcinoma. Adv Exp Med and Biol 1992; 324: 283-93.

8. Shirley SE, Escoffery CT, Sargeant LA, Tulloch T. Clinicopathological features of prostate cancer in Jamaican men. BJU International 2002; 89:390-95.

9. Catalona WJ, Antenor JA, Roehl KA, Moul JW. Screening for prostate cancer in high risk populations. J Urol 2002; 168:1980-84.

10. E. Muñoz, F. Gómez, J.I. Paz, I.Casado, J.M. Silva, M.T. Corcuera, M. J. Alonso. Ki-67 immunolabeling in premalignant lesions and carcinoma of the prostate. Histological correlation and prognostic evaluation. European Journal of histochemistry 2003;47(2):123-28

11. Rashed HE, Kateb M.I, Ragab A, Shaker SS. Evaluation of minimal prostate cancer in needle biopsy specimens using AMACR (P5045), p63 and ki67. Life Sci J 2012; 9(4): 12-21

12. Theodoropoulos VE, Tsigka A, Mihalopoulou A,Tsoukala V, Lazaris AC, Patsouris E. Evaluation of neuroendocrine staining and androgenreceptor expression in incidental prostatic adenocarcinoma: Prognostic implications. Urology 2005;66:897-902.

13. Nilsson S, Nordgren H, Karlberg L, Harvig B, Busch C, Hall $\mathrm{T}$, et al. Expression of estramustine-binding protein (EMBP) and the proliferation-associated antigen $\mathrm{Ki}-67$ in prostatic carcinomas. Scand J Urol Nephrol 1988;110:31-7.

14. Murti K, Warli SM, Laksmi LI. Relations between KI-67 immunohistochemistry expression with histopathology grading and prostate-specific antigen (PSA) values in adenocarcinoma prostate at Dr H. Adam Malik Hospital, Medan Indonesia. Bali Med J 2017; 6(2):289-293

15. Thompson SJ, Mellon K, Charlton RG, Marsh C, Robinson M, Neal DE. P53 and Ki-67 immunoreactivity in human prostate cancer and benign hyperplasia. Br J Urol. 1992; 69:609-13.

*Corresponding author:

Dr. Roopa Urs A.N, Department of Pathology, Mahatma Gandhi Medical College and Research Institute, Pillaiyarakuppam, Puducherry 607402, India Phone: $+918884141327,8248124351$

Email: roopaurspr@gmail.com

Financial or other Competing Interests: None. 